\title{
Formal Concept Analysis and structures underlying Quantum Logics`
}

\author{
O. Krídlo and M. Ojeda-Aciego \\ 1 University of Pavol Jozef Šafárik, Košice, Slovakia \\ 2 Universidad de Málaga. Departamento de Matemática Aplicada. Spain
}

\begin{abstract}
A Hilbert space $H$ induces a formal context, the Hilbert formal context $\bar{H}$, whose associated concept lattice is isomorphic to the lattice of closed subspaces of $H$. This set of closed subspaces, denoted $\mathcal{C}(H)$, is important in the development of quantum logic and, as an algebraic structure, corresponds to a so-called "propositional system", that is, a complete, atomistic, orthomodular lattice which satisfies the covering law. In this paper, we continue with our study of the Chu construction by introducing the Chu correspondences between Hilbert contexts, and showing that the category of Propositional Systems, PropSys, is equivalent to the category of $\mathrm{ChuCors} \mathcal{H}$ of $\mathrm{Chu}$ correspondences between Hilbert contexts.
\end{abstract}

Keywords: Formal Concept Analysis; Chu Correspondence; Quantum Logic

\section{Introduction}

Since its introduction, quantum mechanics raised profound conceptual problems, among which the principal was to find an adequate formalization. John von Neumann [12] investigated mathematical mechanism underlying quantum mechanics, leading two the use of Hilbert spaces as its most natural language. Later, Birkhoff and von Neumann [5] studied the logical structure which is likely to be found in physical theories and, in particular, in quantum mechanics; this led to the discovery that the formalism based on Hilbert spaces also had a (nonclassical) logical structure, which nowadays is called quantum logic. The main conclusion of Birkhoff and von Neumann was

... that one can reasonably expect to find a calculus of propositions which is formally indistinguishable from the calculus of linear subspaces with respect to set products, linear sums, and orthogonal complementsand resembles the usual calculus of propositions with respect to and, or, and not. (Quoted from [5])

\footnotetext{
* Partially supported by Universidad de Mlaga. Campus de Excelencia Internacional Andalucía Tech.
} 
Since then, a number of researchers have continued this line of research, and found alternative descriptions of different interesting subsets of linear subspaces. For instance, in quantum logic, ortholattices have often been used, where the (topologically) closed subspaces of a separable Hilbert space represent quantum propositions; these spaces can be represented in purely lattice-theoretical terms, by Piron's representation theorem [13, as irreducible, complete, atomistic, orthomodular lattices satisfying the covering law. Thus, it makes sense that these special types of lattices are called propositional systems in [14.

For the purposes of this work, we will use an additional characterization, in which closed subspaces turn out to be algebraically characterized in terms of the double orthogonal operator ()$^{\perp \perp}$, i.e., a linear subspace of a Hilbert space is (topologically) closed if and only if is a fixpoint of ()$^{\perp \perp}$.

The lattice of closed subspaces of a Hilbert space has a solid relationship with Formal Concept Analysis (FCA), as stated in [8, page 55], where one can read that

if $H$ is a Hilbert space and $\perp$ is the orthogonality relation, then the concept lattice of the context $(H, H, \perp)$ is isomorphic to the orthomodular lattice of the closed subspaces of $H$. (Quoted from [8])

In this paper, we continue our research line on the Chu construction [6] applied to different generalizations of FCA 910. It is worth to note that the closely related notion of Chu space has already been applied to represent quantum physical systems and their symmetries 12 .

Specifically, the goal of this work is to highlight the importance of the Chu construction with respect to quantum logic, by constructing a category on Hilbert formal contexts $(H, H, \perp)$ and Chu correspondences between them, and proving that it is equivalent to the category PropSys of propositional systems of Hilbert spaces.

The structure of this paper is the following: in Section 2 preliminary notions related to Hilbert spaces, lattice-theoretical approach to the subset of closed linear subspaces, and formal concept analysis are introduced; then, in Section 3 , we give the contextual representation of propositional systems in terms of Hilbert formal contexts and Chu correspondences; finally, in Section 4 we draw some conclusions and present some prospects for future work.

\section{Preliminary definitions}

In this section, we introduce some basic notions about Hilbert spaces, lattices, and formal concept analysis.

\subsection{Hilbert spaces}

Definition 1. A Hilbert space $H$ is a real or complex vector space with an inner product which is also a complete metric space with respect to the distance function induced by the inner product. 
The induced metric topology allows to talk about the closed linear subspaces of $H$. The set of closed linear subspaces of $H$ will be denoted by $\mathcal{C}(H)$.

The inner product in a Hilbert space naturally induces the orthogonality relation: two vectors $v, w \in H$ are orthogonal, written $v \perp w$, if their inner product is zero.

Definition 2. Let $H$ be a Hilbert space, let $S \subseteq H$ be a subspace of $H$ and $v \in H$. We write $v \perp S$ if and only if $v \in S^{\perp}$ or, in other words, $v \perp u$ for all $u \in S$.

It turns out that topological closure of a linear subspace can be directly rephrased in terms of the orthogonality relation, as stated below:

Lemma 1. Let $A \subseteq H$ be a linear subspace of $H$, then $A$ is closed if and only if $A=A^{\perp \perp}$.

Definition 3. A ray in a Hilbert space $H$ is any one-dimensional linear subspace of $H$. Let $u \in H$ be an arbitrary vector of $H$, then we denote

$$
\rho(u)=\{v \in H \mid v=\lambda u \text { for some scalar } \lambda .
$$

The set of all rays in $H$ is denoted by $\mathcal{P}(H)$, whereas the set of all linear subspaces of $H$ is denoted by $\mathcal{L}(H)$.

It is worth to note that, although the main interest of Hilbert spaces is on the merging of algebraic (since it is a vector space) and topological properties (since the inner product induces the metric topology), the main tools used in this paper belong almost exclusively to linear algebra.

\subsection{Lattices}

A lattice $L$ is said to be:

- complete if suprema and infima exist for any subset of $L$.

- atomistic if every element of $L$ is the join of finitely many atoms.

- orthomodular if it has zero element 0 and unit element 1 , and for any element $a$ there is an orthocomplement $a^{\perp}$, i.e. an element satisfying

1. $a \vee a^{\perp}=1, \quad a \wedge a^{\perp}=0, \quad\left(a^{\perp}\right)^{\perp}=a$

2. $a \leq b$ implies $a^{\perp} \geq b^{\perp}$

3. $a \leq b$ implies $b=a \vee\left(b \wedge a^{\perp}\right) \quad$ (orthomodular law)

Definition 4. A propositional system is a complete, atomistic, orthomodular lattice $\left(L, \leq,()^{\perp}\right)$ which satisfies the Covering Law, i.e., for any $x \in L$ and any atom $a \in L$ we have that $a \wedge x=0$ implies $x \prec a \vee x$.

Lemma 2. The algebraic structure of the set $\mathcal{C}(H)$ of closed linear subspaces of a Hilbert space $H$ it that of a propositional system.

Definition 5. Let $C_{1}$ and $C_{2}$ be propositional systems. A map $h: C_{1} \rightarrow C_{2}$ is a morphism of propositional systems if it preserves arbitrary suprema and maps atoms of $C_{1}$ to atoms or the bottom element of $C_{2}$.

Proposition 1. Propositional systems and their morphisms forms a category PropSys. 


\subsection{FCA and intercontextual structures}

Definition 6. Formal context is a triple $(B, A, R)$ where $R \subseteq B \times A$. Lets define two mappings $(-)^{\uparrow}: 2^{B} \rightarrow 2^{A}$ and $(-)^{\downarrow}: 2^{A} \rightarrow 2^{B}$ as follows

$-X^{\uparrow}=\{a \in A \mid(\forall b \in X)(b, a) \in R\}$

- $Y^{\downarrow}=\{b \in B \mid(\forall a \in Y)(b, a) \in R\}$

for any $X \subseteq B$ and $Y \subseteq A$. Such mappings are called derivation (conceptforming) operators of the context $(B, A, R)$.

Proposition 2. Pair of mappings $\left((-)^{\uparrow},(-)^{\downarrow}\right)$ forms a Galois connection between $\left(2^{B}, \subseteq\right)$ and $\left(2^{A}, \subseteq\right)$.

Definition 7. Let $C=(B, A, R)$ be a formal context. A pair of sets $(X, Y) \in$ $2^{B} \times 2^{A}$ is a formal concept if $X^{\uparrow}=Y$ and $Y^{\downarrow}=X$. The object part $X$ is said to be the extent, and the attribute part $Y$ is the intent of the formal concept. The set of all extents and intents of a formal context $C$ are denoted by $\operatorname{Ext}(C)$ and $\operatorname{Int}(C)$, respectively.

Formal concepts $(X, Y)$ are fixpoints of Galois connection $\left((-)^{\uparrow},(-)^{\downarrow}\right)$, i.e. pairs of closed subsets made by closure operators of derivation operators.

We now recall the basic notions about the category ChuCors of formal contexts and Chu correspondences.

Definition 8. Let $C_{1}=\left(B_{1}, A_{1}, R_{1}\right)$ and $C_{2}=\left(B_{2}, A_{2}, R_{2}\right)$ be two formal contexts with pairs derivation operators $\left((-)^{\uparrow_{1}},(-)^{\downarrow_{1}}\right)$ and $\left((-)^{\uparrow_{2}},(-)^{\downarrow_{2}}\right)$. Lets define the following pair of mappings $\varphi=\left(\varphi_{L}, \varphi_{R}\right)$ from $C_{1}$ to $C_{2}$ with denotation $\varphi: C_{1} \rightarrow C_{2}$

$-\varphi_{L}: B_{1} \rightarrow \operatorname{Ext}\left(C_{2}\right)$ and $\varphi_{R}: A_{2} \rightarrow \operatorname{Int}\left(C_{1}\right)$

- and for any $\left(b_{1}, a_{2}\right) \in B_{1} \times A_{2}$ holds that

$$
a_{2} \in \varphi_{L}\left(b_{1}\right)^{\uparrow_{2}} \Longleftrightarrow b_{1} \in \varphi_{R}\left(a_{2}\right)^{\downarrow_{1}}
$$

with composition of $\varphi_{1}: C_{1} \rightarrow C_{2}$ and $\varphi_{2}: C_{2} \rightarrow C_{3}$ defined as follows

$$
\begin{aligned}
& \text { - }\left(\varphi_{1} \circ \varphi_{2}\right)_{L}\left(b_{1}\right)=\left(\bigcup_{b_{2} \in \varphi_{1 L}\left(b_{1}\right)} \varphi_{2 L}\left(b_{2}\right)\right)^{\downarrow_{3} \uparrow_{3}} \\
& \text { - }\left(\varphi_{1} \circ \varphi_{2}\right)_{R}\left(a_{3}\right)=\left(\bigcup_{a_{2} \in \varphi_{2 R}\left(a_{3}\right)} \varphi_{1 R}\left(a_{2}\right)\right)^{\uparrow_{1} \downarrow_{1}}
\end{aligned}
$$

and with identity Chu correspondence $\iota: C \rightarrow C$ for any $C=(B, A, R)$ with derivation operators $\left((-)^{\uparrow},(-)^{\downarrow}\right)$

$$
\begin{aligned}
& \text { - } \iota_{L}(b)=(\{b\})^{\downarrow \uparrow} \text { for any } b \in B \\
& \text { - } \iota_{R}(a)=(\{a\})^{\uparrow \downarrow} \text { for any } a \in A
\end{aligned}
$$

Such pair of mappings $\varphi=\left(\varphi_{L}, \varphi_{R}\right)$ is Chu correspondence. The set of all Chu correspondences from $C_{1}$ to $C_{2}$ is denoted by $\operatorname{ChuCors}\left(C_{1}, C_{2}\right)$.

Theorem 1 ([1] ). Formal contexts and Chu correspondences form a category ChuCors, which is equivalent to the category of complete lattices and supremum preserving maps. 


\section{Contextual representation of propositional systems}

\subsection{Hilbert formal contexts and Hilbert-Chu correspondences}

It is known that if $H$ is a Hilbert space and $\perp$ is the orthogonality relation, then the concept lattice of the context $(H, H, \perp)$ is isomorphic to the orthomodular lattice of the closed subspaces of $H$, since $\left(U, U^{\perp}\right)$ is a concept for each such subspace $U$, see [ㅁ, page 55]. This justifies the following definition:

Definition 9. Let $H$ be a Hilbert space, the tuple $\bar{H}=(H, H, \perp)$ is said to be the Hilbert formal context associated to $H$.

Lemma 3. Let $H$ be a Hilbert space, the concept lattice associated to the Hilbert formal context $\bar{H}$ is a propositional system.

Proof. Follows from Proposition 2 and the construction of the concept lattice of $\bar{H}$.

Lemma 4. Let $H$ be a Hilbert space. Then, the trivial subspace $\{\overline{0}\}$ and the one-dimensional subspaces of $H$ are closed under $(-)^{\perp}$.

Proof. The first statement is trivial.

Given an arbitrary vector $u \in H$, we have to prove that $\rho(u)=\rho(u)^{\perp \perp}$. It is not straightforward to check that $\rho(u) \subseteq \rho(u)^{\perp \perp}$. Now, assume that there exists $v \in H$ such that $v \in \rho(u)^{\perp \perp}$ and $v \notin \rho(u)$; from $v \notin \rho(u)$ it follows that $v$ is not a multiple of $u$, and from $v \in \rho(u)^{\perp \perp}$ we obtain that $v \notin \rho(u)^{\perp}$, therefore there should exist some non-null vector $w \in H$ such that $u \perp w$ and $v \not \perp w$. On the other hand, $v \in \rho(u)^{\perp \perp}$ is equivalent to $v \perp \rho(u)^{\perp}$ that means that everything orthogonal to $\rho(u)$ is orthogonal to $v$ that contradicts to existence of vector $w$.

Definition 10. The category $\mathrm{ChuCors}_{\mathcal{H}}$ has Hilbert formal contexts as objects and, given two Hilbert spaces $H_{1}$ and $H_{2}$, the morphisms between the corresponding Hilbert formal contexts are pairs of mappings $\varphi=\left(\varphi_{L}, \varphi_{R}\right)$ where $\varphi_{L}: H_{1} \rightarrow \mathcal{P}\left(H_{2}\right) \cup\{\overline{0}\}, \varphi_{R}: H_{2} \rightarrow \mathcal{C}\left(H_{1}\right)$ satisfying

$$
\varphi_{L}\left(v_{1}\right) \perp v_{2} \Longleftrightarrow v_{1} \perp \varphi_{R}\left(v_{2}\right)
$$

Note that $\varphi_{L}$ is well-defined since the rays and the trivial subspace are closed and, hence, extents by Lemma 4 furthermore, the image of $\varphi_{R}$ is also closed by construction and, hence, an intent.

Lemma 5. Any $\varphi_{L} \in \mathrm{ChuCors}\left(\overline{H_{1}}, \overline{H_{2}}\right)$ preserves linear dependence of vectors.

Proof. Consider $u_{i}, v_{i}, w_{i} \in H_{i}$ for $i \in\{1,2\}$ and let us write $\rho_{i}(-)$ for the "ray" operator defined on $H_{i}$. Assume that $w_{1}=\alpha_{1} u_{1}+\beta_{1} v_{1}$ for some scalars $\alpha_{1}, \beta_{1}$, and that $\varphi_{L}\left(u_{1}\right)=\rho_{2}\left(u_{2}\right), \varphi_{L}\left(v_{1}\right)=\rho_{2}\left(v_{2}\right)$ and $\varphi_{L}\left(w_{1}\right)=\rho_{2}\left(w_{2}\right)$ for some $u_{2}, v_{2}, w_{2} \in H_{2}$ (since the cases in which some of the images is the $\{\overline{0}\}$ subspace 
is trivial). Now we would like to prove that there exist scalars $\alpha_{2}, \beta_{2}$ such that $w_{2}=\alpha_{2} u_{2}+\beta_{2} v_{2}$.

By reductio ad absurdum, let us assume that $w_{2} \neq \alpha_{2} u_{2}+\beta_{2} v_{2}$, for any $\alpha_{2}, \beta_{2} \in K_{2}$. This means that there should exist $q_{2} \in H_{2}$ such that $q_{2} \perp u_{2}$, $q_{2} \perp v_{2}$ and $q_{2} \not \perp w_{2}$, that is, $q_{2} \perp \varphi_{L}\left(u_{1}\right), q_{2} \perp \varphi_{L}\left(v_{1}\right)$ and $q_{2} \not \perp \varphi_{L}\left(w_{1}\right)$. From Definition 10. we would obtain $u_{1} \perp \varphi_{R}\left(q_{2}\right), v_{1} \perp \varphi_{R}\left(q_{2}\right)$ and $w_{1} \not \perp \varphi_{R}\left(q_{2}\right)$ contradicting the fact that $w_{1}$ depends linearly from $u_{1}$ and $v_{1}$.

Lemma 6. Given two Hilbert spaces $H_{1}, H_{2}$ and $\varphi \in \operatorname{ChuCors}\left(H_{1}, H_{2}\right)$, consider $u, v \in H_{1}$ satisfying $u=\lambda v$ for some $\lambda \in K_{1}$. Then $\varphi(u)=\varphi(v)$.

Proof. We have $\varphi_{L}(u)=\varphi_{L}(v)$ straightforwardly from Lemma 5

Now, if $\varphi_{R}(u) \neq \varphi_{R}(v)$ then there would exist $q \in H_{1}$ such that $q \perp \varphi_{R}(u)$ and $q \not \perp \varphi_{R}(v)$; this is equivalent to $\varphi_{L}(q) \perp u$ and $\varphi_{L}(q) \not \perp v$, which is not possible since $u=\lambda v$.

Corollary 1. By the previous Lemma, we can see any left side $\varphi_{L}$ of any Hilbert-Chu correspondence $\varphi: \overline{H_{1}} \rightarrow \overline{H_{2}}$ as a mapping between atoms (rays) of Hilbert space $H_{1}$ and atoms (rays) or bottom (trivial subspace) of $H_{2}$.

Lemma 7. Let $\overline{H_{1}}, \overline{H_{2}}, \overline{H_{3}}$ be Hilbert formal contexts, and consider two Chu correspondences $\varphi_{1} \in \mathrm{ChuCors}_{\mathcal{H}}\left(\overline{H_{1}}, \overline{H_{2}}\right)$ and $\varphi_{2} \in \operatorname{ChuCors}_{\mathcal{H}}\left(\overline{H_{2}}, \overline{H_{3}}\right)$. Consider the following mappings

$\left(\varphi_{2} \varphi_{1}\right)_{L}\left(v_{1}\right)=\left(\bigcup_{v_{2} \in \varphi_{1 L}\left(v_{1}\right)} \varphi_{2 L}\left(v_{2}\right)\right)^{\perp \perp} ;\left(\varphi_{2} \varphi_{1}\right)_{R}\left(v_{3}\right)=\left(\bigcup_{v_{2} \in \varphi_{2 R}\left(v_{3}\right)} \varphi_{1 R}\left(v_{2}\right)\right)^{\perp \perp}$

then $\left(\left(\varphi_{2} \varphi_{1}\right)_{L},\left(\varphi_{2} \varphi_{1}\right)_{R}\right) \in \operatorname{ChuCors}_{\mathcal{H}}\left(\overline{H_{1}}, \overline{H_{3}}\right)$.

Proof. Firstly, note that by Lemma 5 , the union $\bigcup_{v_{2} \in \varphi_{1 L}\left(v_{1}\right)} \varphi_{2 L}\left(v_{2}\right)$ is closed because, indeed, it is a ray given by the value of any $\varphi_{2 L}\left(v_{2}\right)$ (all of them coincide). The reason to give the definition as the double orthogonal of the union is to clarify that, actually, we are just applying the usual composition of Chu correspondences. For the right part, its definition is the closure (double orthogonal) of certain set and, hence, is closed.

For the proof of the Chu equivalence, consider the following equivalences: 


$$
\begin{aligned}
v_{1} \in\left(\left(\varphi_{2} \varphi_{1}\right)_{R}\left(v_{3}\right)\right)^{\perp} & \Longleftrightarrow v_{1} \in\left(\bigcup_{v_{2} \in \varphi_{2 R}\left(v_{3}\right)} \varphi_{1 R}\left(v_{2}\right)\right)^{\perp \perp \perp}=\left(\bigcup_{v_{2} \in \varphi_{2 R}\left(v_{3}\right)} \varphi_{1 R}\left(v_{2}\right)\right)^{\perp} \\
& \Longleftrightarrow v_{1} \perp u_{1} \text { for all } u_{1} \in \bigcup_{v_{2} \in \varphi_{2 R}\left(v_{3}\right)} \varphi_{1 R}\left(v_{2}\right) \\
& \Longleftrightarrow v_{1} \perp u_{1} \text { for all } v_{2} \in \varphi_{2 R}\left(v_{3}\right) \text { and for all } \forall u_{1} \in \varphi_{1 R}\left(v_{2}\right) \\
& \Longleftrightarrow v_{1} \in \varphi_{1 R}\left(v_{2}\right)^{\perp} \text { for all } v_{2} \in \varphi_{2 R}\left(v_{3}\right) \\
& \Longleftrightarrow v_{2} \in \varphi_{1 L}\left(v_{1}\right)^{\perp} \text { for all } v_{2} \in \varphi_{2 R}\left(v_{3}\right) \\
& \Longleftrightarrow v_{2} \perp u_{2} \text { for all } v_{2} \in \varphi_{2 R}\left(v_{3}\right) \text { and for all } u_{2} \in \varphi_{1 L}\left(v_{1}\right) \\
& \Longleftrightarrow u_{2} \in \varphi_{2 R}\left(v_{3}\right)^{\perp} \text { for all } u_{2} \in \varphi_{1 L}\left(v_{1}\right) \\
& \Longleftrightarrow v_{3} \in \varphi_{2 L}\left(u_{2}\right)^{\perp} \text { for all } u_{2} \in \varphi_{1 L}\left(v_{1}\right) \\
& \Longleftrightarrow v_{3} \perp u_{3} \text { for all } u_{2} \in \varphi_{1 L}\left(v_{1}\right) \text { and for all } u_{3} \in \varphi_{2 L}\left(u_{2}\right) \\
& \Longleftrightarrow v_{3} \perp u_{3} \text { for all } u_{3} \in \bigcup_{u_{2} \in \varphi_{1 L}\left(v_{1}\right)} \varphi_{2 L}\left(u_{2}\right) \\
& \Longleftrightarrow v_{3} \in\left(\bigcup_{u_{2} \in \varphi_{1 L}\left(v_{1}\right)} \varphi_{2 L}\left(u_{2}\right)\right)^{\perp}=\left(\bigcup_{u_{2} \in \varphi_{1 L}\left(v_{1}\right)} \varphi_{2 L}\left(u_{2}\right)\right) \\
& \Longleftrightarrow v_{3} \in\left(\left(\varphi_{2} \varphi_{1}\right)_{L}\left(v_{1}\right)\right)^{\perp}
\end{aligned}
$$

Lemma 8. Composition is associative.

Proof. Associativity of composition is proved in [1]. New definition has no impact on composition. The construction is the same.

Lemma 9. Let $H$ be a Hilbert space and $S \in \mathcal{C}(H)$ arbitrary. If $u \in S$ then $\rho(u) \subseteq S$.

Proof. $\{u\} \subseteq S$ and $\{u\}^{\perp \perp}=\rho(u) \subseteq S^{\perp \perp}=S$.

Lemma 10. There exist a unit morphism $\iota^{H}$ for any object $H$ in $\operatorname{ChuCors}_{\mathcal{H}}$ which is a neutral element of arrow composition.

Proof. Given a Hilbert formal context $H$, the unit morphism is defined by $\iota=$ $\left(\iota_{L}, \iota_{R}\right)$ where $\iota_{L}(v)=\iota_{R}(v)=\{v\}^{\perp \perp}=\rho(v)$ forall $v \in H$. It is easy to check that $\iota \in \mathrm{ChuCors}_{\mathcal{H}}$ : it is indeed a morphism, since $\iota_{L}(v): H \rightarrow \mathcal{P}(H)$, and $\iota_{R}(v): H \rightarrow \mathcal{C}(H)$ and, moreover,

$$
u \perp \iota_{L}(v) \Longleftrightarrow u \perp\{v\}^{\perp \perp} \Longleftrightarrow u \perp v \Longleftrightarrow\{u\}^{\perp \perp} \perp v \Longleftrightarrow \iota_{R}(u) \perp v
$$


Consider $\varphi \in \operatorname{ChuCors}_{\mathcal{H}}\left(H_{1}, H_{2}\right)$ and $\iota_{i}: H_{i} \rightarrow H_{i}$ for $i \in\{1,2\}$. Given, $v_{1} \in H_{1}$, by Lemma 5. we have that $\varphi_{L}(u)=\varphi_{L}\left(v_{1}\right)$ for all $u$ in the ray $\rho\left(v_{1}\right)$ (which coincides with $\iota_{1 L}\left(v_{1}\right)$ ). Then, we have

$$
\begin{gathered}
\left(\iota_{1} \circ \varphi\right)_{L}\left(v_{1}\right)=\left(\bigcup_{u_{1} \in \iota_{1}\left(v_{1}\right)} \varphi_{L}\left(u_{1}\right)\right)^{\perp \perp}=\varphi_{L}\left(v_{1}\right)^{\perp \perp}=\varphi_{L}\left(v_{1}\right) \\
\left(\varphi \circ \iota_{2}\right)_{L}\left(v_{1}\right)=\left(\bigcup_{u_{2} \in \varphi_{L}\left(v_{1}\right)} \iota_{2 L}\left(u_{2}\right)\right)^{\perp \perp}=\left(\bigcup_{u_{2} \in \varphi_{L}\left(v_{1}\right)} \rho\left(u_{2}\right)\right)^{\perp \perp}=\left(\varphi_{L}\left(v_{1}\right)\right)^{\perp \perp}=\varphi_{L}\left(v_{1}\right)
\end{gathered}
$$

Hence $(\iota \circ \varphi)_{L}\left(v_{1}\right)=\varphi_{L}\left(v_{1}\right)=(\varphi \circ \iota)_{L}\left(v_{1}\right)$ for any $v_{1} \in H_{1}$. Now, similarly,

$$
\begin{aligned}
&\left(\iota_{1} \circ \varphi\right)_{R}\left(v_{2}\right)=\left(\bigcup_{u_{1} \in \varphi_{R}\left(v_{2}\right)} \iota_{1 R}\left(u_{1}\right)\right)^{\perp \perp}=\left(\bigcup_{u_{1} \in \varphi_{R}\left(v_{2}\right)} \rho\left(u_{1}\right)\right)^{\perp \perp}=\left(\varphi_{R}\left(v_{2}\right)\right)^{\perp \perp}=\varphi_{R}\left(v_{2}\right) \\
&\left(\varphi \circ \iota_{2}\right)_{L}\left(v_{2}\right)=\left(\bigcup_{u \in \iota_{2 R}\left(v_{2}\right)} \varphi_{R}(u)\right)^{\perp \perp}=\left(\bigcup_{u \in \rho_{2}\left(v_{2}\right)} \varphi_{R}(u)\right)^{\perp \perp} \\
& \text { due to Lemma } 6\left(\bigcup_{u \in \rho\left(v_{2}\right)} \varphi_{R}\left(v_{2}\right)\right)^{\perp \perp}=\left(\varphi_{R}\left(v_{2}\right)\right)^{\perp \perp}=\varphi_{R}\left(v_{2}\right)
\end{aligned}
$$

Hence $\left(\iota \circ \varphi_{R}\right)\left(v_{2}\right)=\varphi_{R}\left(v_{2}\right)=(\varphi \circ \iota)_{R}\left(v_{2}\right)$ for any $v_{2} \in H_{2}$, and the unit arrow $\iota$ is the neutral element for composition of arrows.

As a consequence of the previous results, we obtain

Proposition 3. ChuCors $\mathcal{H}$ forms a category.

\subsection{Connection to PropSys}

In this section we will describe the functorial relation between the categories $\mathrm{ChuCors}_{\mathcal{H}}$ and PropSys. The construction is as follows:

Definition 11. The functor $\Pi:$ ChuCors $\mathcal{H} \rightarrow$ PropSys is defined by:

- Given a Hilbert formal context $\bar{H}$, we define $\Pi(\bar{H})$ as the concept lattice of $\bar{H}$. 
- Let $\varphi$ be a morphism in $\operatorname{ChuCors}_{\mathcal{H}}\left(\overline{H_{1}}, \overline{H_{2}}\right)$. Then $\varphi$ induces a mapping $\bar{\varphi}: \mathcal{P}\left(H_{1}\right) \rightarrow \mathcal{P}\left(H_{2}\right) \cup\{\overline{0}\}$, defined by $\bar{\varphi}(\rho(v))=\varphi_{L}(v)$ for all $v \in H_{1}$ and ray $\rho(v) \in \mathcal{P}\left(H_{1}\right)$. Now, $\Pi(\varphi)$ is defined as the homomorphic extension of $\bar{\varphi} \mathrm{S}^{3}$

The following lemma proves that $\Pi$ is, indeed, a functor.

Lemma 11. $\Pi: \mathrm{ChuCors}_{\mathcal{H}} \rightarrow$ PropSys is a functor.

Proof. Lemma 3 states that $\Pi$ maps objects of ChuCors $\mathcal{H}$ on objects of PropSys.

Consider an arbitrary $\varphi \in \operatorname{ChuCors}_{\mathcal{H}}\left(\overline{H_{1}}, \overline{H_{2}}\right)$. By definition, $\bar{\varphi}$ maps rays of $H_{1}$ (atoms of $\mathcal{C}\left(\mathcal{L}\left(H_{1}\right)\right)$ ) to either rays or zero vector singleton (atoms or bottom of $\left.\mathcal{C}\left(\mathcal{L}\left(H_{2}\right)\right)\right)$ and, moreover, preserves suprema; hence $\Pi$ maps Hilbert-Chu correspondences to morphisms between propositional systems.

Now, $\Pi(\iota)$ is an identity morphism in PropSys, since $\bar{\iota}(\rho(v))=\iota_{L}(v)=\rho(v)$ for all $v \in H$.

Finally, consider $\varphi_{i} \in \operatorname{ChuCors\mathcal {H}}\left(\overline{H_{i}}, \overline{H_{i+1}}\right)$ for $i \in\{1,2\}$, and let us prove that $\Pi\left(\varphi_{1} \circ \varphi_{2}\right)=\Pi\left(\varphi_{1}\right) \circ \Pi\left(\varphi_{2}\right)$. Given $v_{1} \in H_{1}$ we have that

$$
\left(\varphi_{1} \circ \varphi_{2}\right)_{L}\left(v_{1}\right)=\left(\bigcup_{v_{2} \in \varphi_{1 L}\left(v_{1}\right)} \varphi_{2 L}\left(v_{2}\right)\right)^{\perp \perp}
$$

By definition, $\varphi_{1 L}\left(v_{1}\right)$ is either $\{\overline{0}\}$ or a ray of $H_{2}$, and Lemma 6 ensures that for all $u_{2}, w_{2} \in \varphi_{1 L}\left(v_{1}\right)=\overline{\varphi_{1}}\left(\rho\left(v_{1}\right)\right)$ we have $\varphi_{2 L}\left(u_{2}\right)=\varphi_{2 L}\left(w_{2}\right)$, in other words $\overline{\varphi_{2}}\left(\rho\left(u_{2}\right)\right)=\overline{\varphi_{2}}\left(\rho\left(w_{2}\right)\right)$. Hence

$$
\bigcup_{v_{2} \in \overline{\varphi_{1}}\left(\rho\left(v_{1}\right)\right)} \overline{\varphi_{2}}\left(\rho\left(v_{2}\right)\right)=\overline{\varphi_{2}}\left(\overline{\varphi_{1}}\left(\rho\left(v_{1}\right)\right)\right)
$$

We recall now some necessary notions which will be used in order to prove that the previous functor satisfies the conditions to define a categorical equivalence.

\section{Definition 12.}

1. A functor $F: \mathcal{C} \longrightarrow \mathcal{D}$ is faithful if for all objects $A, B$ of a category $\mathcal{C}$, the map $F_{A, B}: \operatorname{Hom}_{\mathcal{C}}(A, B) \longrightarrow \operatorname{Hom}_{\mathcal{D}}(F(A), F(B))$ is injective.

2. Similarly, $F$ is full if $F_{A, B}$ is always surjective.

The proof of the categorical equivalence will be done by using the following characterization:

Theorem 2 (See [3]). The following conditions on a functor $F: \mathcal{C} \longrightarrow \mathcal{D}$ are equivalent:

\footnotetext{
${ }^{3}$ We will abuse the notation and write $\bar{\varphi}$ to refer to this homomorphic extension.
} 
- $F$ is an equivalence of categories.

- $F$ is full and faithful and "essentially surjective" on objects: for every $D \in \mathcal{D}$ there is some $C \in \mathcal{C}$ such that $F(C) \cong D$.

Theorem 3. Categories ChuCors $\mathcal{H}$ and PropSys are equivalent.

Proof. By the definition of ChuCors ${ }_{\mathcal{H}}$ and $\Pi$ is not difficult to see that the mapping $\Pi\left(H_{1}, H_{2}\right):$ ChuCors $\mathcal{H}\left(\overline{H_{1}}, \overline{H_{2}}\right) \rightarrow \operatorname{PropSys}\left(\Pi\left(\overline{H_{1}}\right), \Pi\left(\overline{H_{2}}\right)\right)$ is bijective. Hence $\Pi$ is full and faithful.

Let $\mathcal{L}=\left(L, \leq,(-)^{\perp}\right)$ be an arbitrary propositional system. Essential surjectivity of functor $\Pi$ means that there exist a Hilbert formal context whose concept lattice is isomorphic to $\mathcal{L}$.

Let $L_{a t}$ be the set of atoms of $\mathcal{L}$, and let us prove that concept lattice of the formal context $\left(L_{a}, L_{a}, \perp\right)$ where $a \perp b \Longleftrightarrow\left(a \leq b^{\perp}\right) \Longleftrightarrow\left(b \leq a^{\perp}\right)$, for any two atom $a, b \in L_{a t}$, is isomorphic to $\mathcal{L}$. Since $\mathcal{L}$ is atomistic, we have that every element $x \in L$ is the supremum of a finite set of atoms, say $x=\bigvee_{i \in I_{x}} a_{i}$. This means the set of atoms $L_{a t}$ is supremum-dense in $L$. Moreover

$$
x=x^{\perp \perp}=\left(\bigvee_{i \in I_{x}} a_{i}\right)^{\perp \perp}=\left(\bigwedge_{i \in I_{x}} a_{i}^{\perp}\right)^{\perp} .
$$

Furthermore, every element $y \in L$ can be represented as $y=x^{\perp}=\bigwedge_{i \in I_{x}} a_{i}^{\perp}$. Hence the set $\overline{L_{a t}}=\left\{a^{\perp} \mid a \in L_{A}\right\}$ is infimum-dense in $L$. Therefore, by the second part of the basic theorem of concept lattices, the concept lattice of $\left(L_{a t}, \overline{L_{a t}}, \leq\right)$ or $\left(L_{a t}, L_{a t}, \perp\right)$ is isomorphic to $\mathcal{L}$.

\section{Conclusions}

Continuing with the study of generalized Chu correspondences, we have introduced the new category $\mathrm{ChuCors}_{\mathcal{H}}$, whose objects are Hilbert formal contexts and whose morphisms are $\mathrm{Chu}$ correspondences between them. The notion of Hilbert formal context $(H, H, \perp)$ associated to a Hilbert space $H$ was already present in Ganter and Wille's book [8], stating its close relationship with the ortholattice of closed linear subspaces and, hence, to the theory of propositional systems as algebraic structures underlying quantum logic. The main result in this work is the proof that the category $\mathrm{ChuCors}_{\mathcal{H}}$ is equivalent to the category PropSys of propositional systems.

This opens the way to future work oriented to the use of Chu correspondences to analyze more structures related to quantum logics, such as those in Abramsky's big toy models [1].

\section{References}

1. S. Abramsky. Big toy models: representing physical systems as Chu spaces. Synthese, 186(3):697-718, 2012. 
2. S. Abramsky. Coalgebras, Chu spaces, and representations of physical systems. Journal of Philosophical Logic, 42(3):551-574, 2013.

3. S. Awodey. Category Theory, Oxford Univ. Press, 2010

4. M. Barr. *-Autonomous categories, vol. 752 of Lecture Notes in Mathematics. Springer-Verlag, 1979.

5. G. Birkhoff and J. von Neumann. The Logic of Quantum Mechanics, Annals of Mathematics 37(4):823-843, 1936

6. P.-H. Chu. Constructing *-autonomous categories. Appendix to 4, pages 103-107.

7. K. Engesser, D.M. Gabbay and D. Lehmann (eds). Handbook of quantum logic and quantum structures: Quantum structures. Elsevier, 2007

8. B. Ganter and R. Wille. Formal concept analysis. Springer-Verlag. 1999

9. O. Kridlo, S. Krajči and M. Ojeda-Aciego, The category of $L$-Chu correspondences and the structure of $L$-bonds, Fundamenta Informaticae 115(4):297-325, 2012.

10. O. Kridlo and M. Ojeda-Aciego, On the $L$-fuzzy generalization of Chu correspondences, Intl J of Computer Mathematics 88(9):1808-1818, 2011.

11. H. Mori. Chu correspondences. Hokkaido Mathematical Journal, 37:147-214, 2008.

12. J. von Neumann. Mathematical Foundations of Quantum Mechanics, Princeton Univ. Press, 1955. Reprinted from the original version published in 1932.

13. C. Piron. Foundations of Quantum Physics, W. A. Benjamin, Inc., 1976.

14. I. Stubbe and B. Van Steirteghem. Propositional systems, Hilbert lattices and generalized Hilbert spaces. In 7], pp. 477-524, 2007 